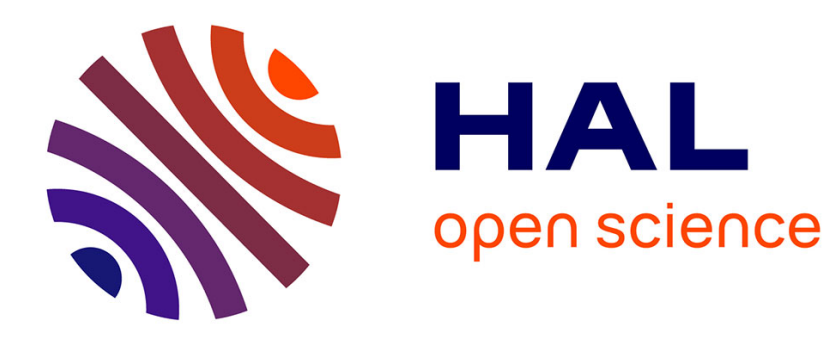

\title{
The equations of motion of a one-velocity, one-temperature bubbly fluid (revisited)
}

\author{
D. Lhuillier
}

\section{To cite this version:}

D. Lhuillier. The equations of motion of a one-velocity, one-temperature bubbly fluid (revisited). Journal de Physique, 1985, 46 (8), pp.1325-1333. 10.1051/jphys:019850046080132500 . jpa-00210076

\section{HAL Id: jpa-00210076 https://hal.science/jpa-00210076}

Submitted on 1 Jan 1985

HAL is a multi-disciplinary open access archive for the deposit and dissemination of scientific research documents, whether they are published or not. The documents may come from teaching and research institutions in France or abroad, or from public or private research centers.
L'archive ouverte pluridisciplinaire HAL, est destinée au dépôt et à la diffusion de documents scientifiques de niveau recherche, publiés ou non, émanant des établissements d'enseignement et de recherche français ou étrangers, des laboratoires publics ou privés. 
Classification

Physics Abstracts

$47.55 \mathrm{~K}-46.20$

\title{
The equations of motion of a one-velocity, one-temperature bubbly fluid (revisited)
}

\author{
D. Lhuillier \\ Laboratoire de Mécanique Théorique $(*)$, Université P. et M. Curie, \\ 4, Place Jussieu, 75230 Paris Cedex 05, France
}

(Reçu le 3 décembre 1984, révisé le 1er avril 1985, accepté le 18 avril 1985)

\begin{abstract}
Résumé. - On présente un modèle, style milieu continu, d'une suspension non diluée de sphères de rayon variable. Dans un souci de simplification et pour mieux insister sur la dynamique du gonflement, nous laissons de côté tous les problèmes concernant le mouvement relatif et la différence de température entre bulles et fluide environnant. On montre qu'au degré de liberté de dilatation est associé le moment scalaire de la quantité de mouvement du mélange, qui joue en quelque sorte le rôle du moment vectoriel en ce qui concerne le degré de liberté de rotation. L'équation du mouvement de cette nouvelle variable fournit une forme non diluée de l'équation de RayleighPlesset. Cette description simplifiée des liquides à bulles fait intervenir six fonctions phénoménologiques de la fraction volumique des bulles. L'une d'entre elles est reliée à la fréquence de résonance des bulles et est accessible par mesure de la vitesse du son. Parmi les cinq fonctions restantes, se trouvent quatre viscosités. On détaille leurs rôles respectifs et on corrige une erreur tenace concernant la viscosité en volume des liquides à bulles.
\end{abstract}

\begin{abstract}
We present a continuum model for a non-dilute suspension of spheres with variable radius. To simplify the resulting equations and to stress the expansion dynamics only, we leave aside the problems of relative motion and temperature difference between the spheres and the surrounding liquid. It is shown that the state variable associated with the expansion degree of freedom is the scalar moment of momentum of the whole mixture, which is somewhat the analog of the usual angular momentum for the rotational degree of freedom. The equation of motion of this new variable leads to a non-dilute form of the Rayleigh-Plesset equation. Our simplified description of bubbly fluids involves six phenomenological functions of the bubble volume fraction. One of them is linked to the bubble resonant frequency and is attainable by sound velocity measurements. Among the five remaining functions are four viscosities. We detail their respective roles and correct a long-lived erroneous statement concerning the bulk or second viscosity of the bubbly fluid.
\end{abstract}

\section{Introduction.}

Bubbly fluids represent an important class of dispersions with deformable particles, and for many industrial purposes, it would be interesting to have a good continuum description of such a medium. Early studies were performed by Rayleigh [1,2] who proposed his famous equation for the evolution in time of the bubble radius, and also by Taylor $[3,4]$ who calculated the apparent bulk viscosity of the mixture. These pioneering contributions were concerned with very dilute systems, and efforts towards a better understanding of concentrated bubbly fluids have developed following two main approaches : the first one, the rigorous approach, involves averaging techniques à

(*) Laboratoire associé au CNRS, L.A. 229. la Batchelor [5, 6] or cell models $[7,8]$ to determine the influence, on the equations of motion, of the hydrodynamic interactions between bubbles. In fact, due to computational difficulties, these methods to date are limited to the $\alpha_{\mathrm{g}}^{2}$ terms, where $\alpha_{\mathrm{g}}$ is the bubble volume fraction. The second or phenomenological approach, is based on the methods of continuum mechanics [9-15] and leads to a set of equations which, in principle, is valid whatever the volume fraction (provided $\alpha_{\mathrm{g}} \lesssim 50 \%$ to have a dispersion). In this approach many phenomenological functions of $\alpha_{\mathrm{g}}$ are introduced but left undetermined. These functions are precisely those partly computed by the rigorous approaches. Thus, both approaches are mutually helpful, somewhat like the kinetic theory, which is a necessary partner of the phenomenological NavierStokes equations when $a b$ initio calculations of the viscosity or heat conduction coefficients are needed. 
The present work belongs to the phenomenological approach. It might at first sight seem surprising that one again looks in this direction, since worthy works already exist. In fact, our motivation is double. First we have the feeling that one can still improve the description of inertia effects in dispersed mixtures; examples concerning particles rotating in a fluid or moving relative to it, suggest that the variables used up to now to express the bubble expansion are not the most convenient ones. Our second motivation is that with the rigorous approach, we now dispose of some exact results in the dilute limit and the phenomenological models so far developed are compatible with many but not all of these results.

Our aim is thus to build a phenomenological model which satisfactorily describes the collective effects in a suspension of expanding spheres, and which leads to all known exact results in the dilute limit. In fact we shall here restrict ourselves to a very simplified model where the relative motion as well as the temperature difference between the bubbles and the liquid are neglected. For simplicity and conciseness we want to stress the expansional degree of freedom only.

In section 2 we comment upon the RayleighPlesset equation, review the existing work on bubbly fluids and discuss the concept of scalar moment of momentum. In section 3 we obtain the average thermodynamic relations for the two components of the bubbly fluid. An expression for the kinetic energy which properly takes into account the hydrodynamic interactions is proposed in section 4 and the Gibbs equation, the cornerstone of the present theory, is obtained in section 5. Then follows a discussion concerning the general form of the equation of motion of the state variables, from which stems the entropy production (section 6). Finally, in section 7 we comment on the resulting equations, we determine the sound velocity and its dependence on the volume fraction, and we examine Taylor's result for the bulk viscosity of the mixture.

A point of nomenclature : in what follows, all quantities with a tilde may fluctuate on a scale much smaller than the bubble radius while the others fluctuate on a scale much larger than the interbubble distance, i.e. a tilde means a local value while the absence of tilde means an averaged value.

\section{A few comments on the Rayleigh-Plesset equation.}

2.1 INFLUENCE OF THE BUBBLE INERTIA AND VISCOSITY. - The dynamical effects produced by the expansion or contraction of a spherical bubble of radius $a(t)$ in an incompressible liquid of density $\rho_{1}^{0}$ and viscosity $\eta_{1}$ are described by the Rayleigh-Plesset equation [2]

$\rho_{1}^{0} a \ddot{a}+\frac{3}{2} \rho_{1}^{0} \dot{a}^{2}+4 \eta_{1} \frac{\dot{a}}{a}=\tilde{p}_{\mathrm{gi}}-\tilde{\tau}_{\mathrm{gi}}-p_{1}-\frac{2 \sigma}{a}$

where $\dot{a}=\mathrm{d} a / \mathrm{d} t, \ddot{a}=\mathrm{d}^{2} a / \mathrm{d} t^{2}, p_{1}$ is the liquid pressure far from the bubble ( $p_{1}$ is also the mean liquid pressure since the liquid is of infinite extent), $\sigma$ is the surface tension between the bubble and the liquid, while $\tilde{p}_{\mathrm{gi}}$ is the bubble pressure at the interface and $\tilde{\tau}_{\mathrm{gi}}$ is a short notation for the component $\left(\tilde{\tau}_{g}\right)_{\text {rr }}$ of the bubble stress at the interface. This equation stems from the momentum conservation in the liquid and the boundary conditions at the interface, but it does not involve the details of the bubble internal motion.

Since we are ultimately interested in the average description of a bubbly mixture, it would be interesting to obtain an equation like (1) but involving the mean bubble pressure $p_{\mathrm{g}}$ and the mean bubble stress instead of their values at the interface. A rapid answer to this problem would be to say that, if we neglect all inertia and viscous effects inside the bubble, we merely have

$$
\tilde{p}_{\mathbf{g i}}-\tilde{\tau}_{\mathbf{g i}}=p_{\mathbf{g}} .
$$

Let us go beyond that approximation and obtain a more precise link between $\tilde{p}_{\mathrm{gi}}-\tilde{\tau}_{\mathrm{gi}}$ and the bubble internal motion. The velocity field inside the bubble will be assumed to be a radially homogeneous expansion, that is to say

$$
\widetilde{v}_{\mathrm{g}}=\frac{r}{a} \dot{a}
$$

In this case, if the condition

$$
\frac{a}{\tilde{\rho}_{\mathrm{g}}^{0}} \frac{\partial \tilde{\rho}_{\mathrm{g}}^{0}}{\partial r} \ll 1
$$

is satisfied, the mass per unit volume of gas $\tilde{\rho}_{\mathbf{g}}^{0}$ depends on time only, hence $\tilde{\rho}_{\mathbf{g}}^{0} \equiv \rho_{\mathbf{g}}^{0}$ and

$$
\rho_{\mathrm{g}}^{0}(t) a^{3}(t) \text { is constant } .
$$

With the velocity field (2), the bubble internal stress $\tilde{\underline{\tau}}_{\mathbf{g}}$ is

$$
\underline{\underline{\tilde{\tau}_{\mathrm{g}}}}=3 \xi_{\mathrm{g}} \frac{\dot{a}}{a} \underline{\underline{I}}
$$

where $\xi_{g}$ is the bulk viscosity of the gas. Thus the bubble stress is also a function of time only and its radial component at the interface is

$$
\tilde{\tau}_{\mathrm{gi}}=3 \xi_{\mathrm{g}} \frac{\dot{a}}{a},
$$

while the momentum conservation law for the bubble merely becomes

$$
\frac{\partial \widetilde{p}_{\mathbf{g}}}{\partial r}=-\rho_{\mathrm{g}}^{0} \frac{r}{a} \ddot{a}
$$

Taking (4) into account, we see that the mean value $p_{\mathrm{g}}$ of the bubble pressure is linked to its value $\tilde{p}_{\mathrm{gi}}$ at the interface by

$$
p_{\mathrm{g}}=\widetilde{p}_{\mathrm{gi}}+\frac{1}{5} \rho_{\mathrm{g}}^{0} a \ddot{a}
$$


Another consequence of equation (6) is to rewrite condition (3) in the form

$$
a \ddot{a} \ll C_{\mathrm{g}}^{2}
$$

where $C_{\mathrm{g}}$ is the velocity of sound in the bubble. In other words, provided the radial acceleration of its interface is compatible with (8), a bubble suffering a homogeneous expansion develops internal stresses given by equations (5) and (7), and the RayleighPlesset equation (1) can be rewritten in terms of average quantities as

$$
\begin{array}{r}
\left(\frac{1}{5} \rho_{\mathrm{g}}^{0}+\rho_{1}^{0}\right) a \ddot{a}+\frac{3}{2} \rho_{1}^{0} \dot{a}^{2}+\left(4 \eta_{1}+3 \xi_{\mathrm{g}}\right) \frac{\dot{a}}{a}= \\
=p_{\mathrm{g}}-p_{1}-\frac{2 \sigma}{a} .
\end{array}
$$

Obviously a bubble is such that

$$
\rho_{\mathrm{g}}^{0} \ll \rho_{1}^{0} \quad \text { and } \quad \xi_{\mathrm{g}} \ll \eta_{1}
$$

and the reader may wonder why we insist on introducing the effects of bubble inertia and viscosity. This will be clearer later on, but at this point we can only say that to prove the generality of certain forthcoming results (like equations (11) or (60) for instance), we absolutely need to know the exact role of $\rho_{\mathbf{g}}^{0}$ and $\xi_{\mathbf{g}}$ in the Rayleigh-Plesset equation.

\subsection{A NEW INTERPRETATION OF THE RAYLEIGH-PLESSET} EQUATION. - When the bubble expands (or shrinks) according to (2), the liquid, supposed to be incompressible, acquires a radial velocity field

$$
\tilde{v}_{1}=\frac{a^{2}}{r^{2}} \dot{a} .
$$

The bubble and its surrounding fluid then has a kinetic energy

$$
e_{\mathrm{kin}}=2 \pi\left(\frac{1}{5} \rho_{\mathrm{g}}^{0}+\rho_{1}^{0}\right) a^{3} \dot{a}^{2},
$$

while the internal energy varies with the bubble radius according to

$$
\mathrm{d} u=-4 \pi a^{2}\left(p_{\mathrm{g}}-p_{1}-\frac{2 \sigma}{a}\right) \mathrm{d} a .
$$

Provided the viscous terms are discarded, one can now verify that the extended Rayleigh-Plesset equation (9) can be written in the remarkable form

$$
\frac{\mathrm{d}}{\mathrm{d} t}\left(\frac{\partial e_{\text {kin }}}{\partial v}\right)=a \frac{\partial e_{\text {kin }}}{\partial a}-a \frac{\mathrm{d} u}{\mathrm{~d} a}
$$

where

$$
v=\frac{\dot{a}}{a}
$$

is the expansion rate of the bubble. The above equation represents the equation of motion of what may be called the scalar moment of momentum of the bubble + liquid system. This quantity is defined as

$$
M=\frac{\partial e_{\text {kin }}}{\partial v}=I v
$$

where

$$
I=4 \pi a^{5}\left(\rho_{1}^{0}+\frac{\rho_{\mathrm{g}}^{0}}{5}\right)
$$

is the moment of inertia in the expanding motion. The scalar moment of momentum plays for the expansion motion (2) the same role as the angular moment of momentum

$$
\overrightarrow{\mathbf{M}}=\frac{\partial e_{\text {kin }}}{\partial \vec{\Omega}}
$$

for the rotating motion $\widetilde{\mathbf{v}}_{\mathbf{g}}=\widetilde{\boldsymbol{\Omega}} \times \mathbf{r}$, and it may also be compared to the internal momentum

$$
\mathbf{P}=\frac{\partial e_{\text {kin }}}{\partial \mathbf{w}}
$$

appearing in the motion of the bubble with a velocity $w$ relative to the liquid. In fact the study of the motion of a rigid particle in a perfect fluid has already shown that the equations of motion are most elegantly rewritten using an overall internal momentum or angular momentum $[16,17]$. We have extended these results to deformable spheres [18] and proved the same interesting property for the overall scalar moment of momentum. The argument is as follows :

Let us consider a bubble expanding in a perfect fluid and define its scalar moment of momentum as

$$
M_{\mathrm{g}}=\int_{0}^{a(t)}\left(\tilde{\rho}_{\mathrm{g}}^{0} \tilde{v}_{\mathrm{g}} r\right) 4 \pi r^{2} \mathrm{~d} r .
$$

The evolution in time of $M_{\mathrm{g}}$ can be deduced from the Euler equations and boundary conditions. The result is

$$
\frac{\mathrm{d} M_{\mathrm{g}}}{\mathrm{d} t}=2 e_{\mathrm{kin}}^{\mathrm{g}}+4 \pi a^{3}\left(p_{\mathrm{g}}-\frac{2 \sigma}{a}\right)+a f_{1}
$$

where

$$
e_{\mathrm{kin}}^{\mathrm{g}}=\frac{1}{2} \int_{0}^{a} \tilde{\rho}_{\mathrm{g}}^{0} \widetilde{v}_{\mathrm{g}}^{2} 4 \pi r^{2} \mathrm{~d} r
$$

and

$$
f_{1}=-\int_{S_{\mathrm{b}}} \tilde{p}_{1 \mathrm{i}} \mathrm{d} S
$$

We thus see that the evolution of $M_{\mathrm{g}}$ is linked to the scalar moment of the force exerted by the surrounding fluid $\left(\tilde{p}_{1 i}\right.$ is the liquid pressure at the interface $\left.S_{\mathrm{b}}\right)$. 
Now it was shown in [18] that

$$
a f_{1}=-4 \pi a^{3} p_{1}-\frac{\mathrm{d}}{\mathrm{d} t}\left(\frac{\partial e_{\mathrm{kin}}^{1}}{\partial v}\right)+a \frac{\partial e_{\mathrm{kin}}^{1}}{\partial a}
$$

where

$$
e_{\mathrm{kin}}^{1}=2 \pi \rho_{1}^{0} a^{5} v^{2}
$$

is the kinetic energy of the fluid. Moreover if (and only if) the bubble internal velocity is given by (2), one can show that

$$
\begin{aligned}
& e_{\mathrm{kin}}^{\mathrm{g}}=\frac{2 \pi}{5} \rho_{\mathrm{g}}^{0} a^{5} v^{2}, \\
& M_{\mathrm{g}}=\frac{\partial e_{\mathrm{kin}}^{\mathrm{g}}}{\partial v}=\frac{4 \pi}{5} \rho_{\mathrm{g}}^{0} a^{5} v,
\end{aligned}
$$

and taking (4) into account

$$
2 e_{\mathbf{k i n}}^{\mathrm{g}}=a \frac{\partial e_{\mathbf{k i n}}^{\mathrm{g}}}{\partial a}
$$

In this case, the evolution (14) of the bubble moment of momentum can be rewritten in the compact form (11) since

$$
e_{\mathrm{kin}}=e_{\mathrm{kin}}^{\mathrm{g}}+e_{\mathrm{kin}}^{1} .
$$

As a consequence we may interpret the RayleighPlesset equation : (14));

- either as the evolution equation for $M_{\mathrm{b}}$ (form

- or as the evolution equation for the moment of momentum of the liquid and the bubble (form (11)).

For the continuum model of bubbly fluids that we want to build, the second interpretation is more relevant.

It is remarkable that the concept of a scalar (and even tensorial) moment of momentum already appeared twenty years ago when Eringen [19] proposed his theory for the so-called micromorphic fluid, in which each material point is characterized not only by the usual velocity but also by other dynamical quantities similar to $\overrightarrow{\boldsymbol{\Omega}}$ and $v$. However, the equations proposed by Eringen were based on the hypothesis that the micromotion in each material point was of the type

$$
\tilde{\mathbf{v}}=v \mathbf{r}+\overrightarrow{\mathbf{\Omega}} \times \mathbf{r} .
$$

In fact, as we shall see in the next section, the material " point " in the continuum description of a bubbly fluid is a volume which contains many bubbles together with the liquid. And while the bubbles are likely to have an internal motion like (15) (and (2) is a peculiar example) the liquid motion is certainly quite different. Hence, we conclude that the work based on Eringen's theory and specialized to the micromotion $\tilde{\mathbf{v}}=v \mathbf{r}[9,10]$ can only apply to a collection of spherical bubbles in vacuum. Subsequent theories of bubbly fluids [11, 13, 15], exhaustively reviewed in [20] and presented as a special class of structured mixtures, have used the convective time derivative of the bubble volume fraction, $\dot{\alpha}_{g}$, as a measure of the varying sphere radius. But this description only holds in the absence of any relative velocity between the bubbles and the liquid. In the theory developed by Bedford and Drumheller from a variational principle $[12,14]$, the bubble expansion is measured with the convective time derivative $\dot{\rho}_{\mathrm{g}}^{0}$. Owing to (4) this variable is a good measure of $\dot{a}$ whatever the relative velocity. But this choice is only justified for bubbles of inert gas since for vapour bubbles the expansion $\dot{a}$ is no longer directly linked to $\dot{\rho}_{\mathrm{g}}^{0}$. At variance with $\dot{\alpha}_{\mathrm{g}}$ or $\dot{\rho}_{\mathrm{g}}^{0}$, the scalar moment of momentum is always proportional to the bubble volumetric changes, whatever the situation. It must be understood that our criticisms of the existing literature are centred on the way the bubble expansion is taken into account. Many contributions have thoroughly dealt with bubbly fluids, describing the velocity difference and temperature difference between the bubbles and the liquid. There is no doubt that references like [12] or [21] are of considerable importance for the subject. We just have the feeling that one can substantially improve the parts of these works concerning the expansional degree of freedom, and this is the raison d'être of this paper.

In fact, we shall take advantage of the new interpretation (11) of the Rayleigh-Plesset equation to build a model of bubbly fluids that associates a variable of the structured mixtures, the gas volume fraction, with a variable of the micromorphic fluid, the scalar moment of momentum. We shall see that this association is quite fruitful.

\section{The average thermodynamic relations for the com- ponents in the mixture.}

3.1 THE LIQUID AND THE GAS. - Both the gas inside the bubbles and the surrounding liquid are described by a Gibbs equation

$$
\mathrm{d} \widetilde{\varepsilon}_{\mathbf{k}}=\tilde{T}_{\mathrm{k}} \mathrm{d} \tilde{s}_{\mathrm{k}}-\tilde{p}_{\mathrm{k}} \mathrm{d}\left(\frac{1}{\tilde{\rho}_{\mathrm{k}}^{0}}\right),
$$

and a Gibbs-Duhem relation

$$
\frac{1}{\tilde{\rho}_{\mathbf{k}}^{0}} \mathrm{~d} \tilde{p}_{\mathbf{k}}=\mathrm{d} \tilde{\mu}_{\mathbf{k}}+\tilde{s}_{\mathbf{k}} \mathrm{d} \tilde{T}_{\mathbf{k}}
$$

where the index $\mathbf{k}$ is either 1 for the liquid or $\mathbf{g}$ for the gas. In the above relations $\tilde{\rho}_{\mathbf{k}}^{0}, \tilde{p}_{\mathbf{k}}, \tilde{T}_{\mathbf{k}}, \widetilde{\varepsilon}_{\mathbf{k}}, \widetilde{s}_{\mathbf{k}}$ and $\tilde{\mu}_{\mathbf{k}}$ are respectively the mass per unit volume, pressure, temperature, specific internal energy, specific entropy and chemical potential or free enthalpy per unit mass, i.e.

$$
\tilde{\mu}_{\mathbf{k}}=\tilde{\varepsilon}_{\mathbf{k}}-\tilde{T}_{\mathbf{k}} \tilde{s}_{\mathbf{k}}+\frac{\tilde{p}_{\mathbf{k}}}{\tilde{\rho}_{\mathbf{k}}^{0}}
$$


These thermodynamic relations hold on a scale much smaller than the dimension of a bubble. We are in fact interested in average thermodynamic relations, valid in any volume of the mixture containing many bubbles, but still being a "point " as compared to macroscopic volumes over which the average variables have noticeable gradients. In such a mesoscale volume the liquid variables (pressure, temperature, density, etc...) vary from point to point, and inside the bubbles themselves, the gas variables also have spatial fluctuations. If we may neglect the time fluctuations due to some random motion of the bubbles or to some turbulent motion of the liquid, we must only worry about these spatial fluctuations (or microscopic gradients) to get the average thermodynamic relations.

If the radial acceleration $\ddot{a}$ of the bubble satisfies equation (8) and if the radial velocity $\dot{a}$ is much smaller than the velocity of sound in the liquid, then : i) $\tilde{\rho}_{1}^{0}$ has negligible fluctuations on a scale of the order of the distance between bubbles; ii) inside a given bubble, $\tilde{\rho}_{\mathrm{g}}^{0}$ depends on time only. As a consequence $\tilde{\rho}_{1}^{0} \simeq \rho_{1}^{0}$ and $\tilde{\rho}_{\mathrm{g}}^{0} \simeq \rho_{\mathrm{g}}^{0}$. But it must be understood that the mean values $\rho_{\mathrm{g}}^{8}$ and $\rho_{1}^{0}$ may vary (slightly) from one mesoscale volume to a neighbouring one.

For low enough $\dot{a}$, we may also suppose that the heat exchanges between the bubbles and the fluid are quite large so that $:$ i) despite the growth or collapse of the bubbles, there are no significant temperature gradients inside the bubbles and in the nearby liquid, hence $\tilde{T}_{1}=T_{1}$ and $\tilde{T}_{\mathrm{g}}=T_{\mathrm{g}}$; ii) there is no difference between the mean temperature of the bubbles and that of the liquid, i.e. $T_{\mathrm{g}}=T_{1}=T$. Clearly, the common temperature $T$ may vary (slightly) from a mesoscale volume to a neighbouring one.

In short, for low enough $\dot{a}$ and $\ddot{a}$ and high enough heat exchanges, the spatial fluctuations or microgradients of $\tilde{\rho}_{1}^{0}, \tilde{\rho}_{\mathrm{g}}^{0}, \widetilde{T}_{1}$ and $\widetilde{T}_{\mathrm{g}}$ are negligible in a mesoscale volume. In this case no product of fluctuations appears in the relations (16) to (18), and the averaging procedure in a mesoscale volume is quite simple : we multiply each of these three relations by the function of presence $\chi_{\mathbf{k}}(\mathbf{r}, t)$ (the value of which is 1 or 0 depending on the presence or absence of phase $k$ at point $\mathbf{r}$ and time $t$ ) and we then get three relations containing average values only and involving the probability of presence $\alpha_{k}$ (the average value of $\chi_{\mathbf{k}}$ ). These average relations are

$$
\begin{aligned}
& \mathrm{d}\left(\rho_{\mathbf{k}} \varepsilon_{\mathbf{k}}\right)=\mu_{\mathbf{k}} \mathrm{d} \rho_{\mathbf{k}}+T \mathrm{~d}\left(\rho_{\mathbf{k}} s_{\mathbf{k}}\right)-p_{\mathbf{k}} \mathrm{d} \alpha_{\mathbf{k}}, \\
& \alpha_{\mathbf{k}} \mathrm{d} p_{\mathbf{k}}=\rho_{\mathbf{k}} \mathrm{d} \mu_{\mathbf{k}}+\rho_{\mathbf{k}} s_{\mathbf{k}} \mathrm{d} T,
\end{aligned}
$$

and

where

$$
\rho_{\mathbf{k}} \varepsilon_{\mathbf{k}}+\alpha_{\mathbf{k}} p_{\mathbf{k}}=\rho_{\mathbf{k}} \mu_{\mathbf{k}}+\rho_{\mathbf{k}} s_{\mathbf{k}} T,
$$

$$
T=T_{1}=T_{\mathrm{g}}
$$

is the temperature of the mixture. The above for- mulae are written with the standard variables of the theory of mixtures where, instead of the average value $\rho_{\mathrm{k}}^{0}$ of the mass per unit volume of pure phase $\mathrm{k}$, one prefers the mass per unit volume of the mixture :

$$
\rho_{\mathrm{k}}=\alpha_{\mathbf{k}} \rho_{\mathrm{k}}^{0}
$$

3.2 The INTERFACE. - To take the surface tension into account, the interface may be thought of as a third phase. The only difference with the liquid or the gas is that the pressure of the interface is negative and that one can arrange for this phase to be massless. The resulting average thermodynamic relations, to be compared with (19) to (21), are

$$
\begin{aligned}
\mathrm{d} U_{\mathrm{a}} & =T_{\mathrm{i}} \mathrm{d} S_{\mathrm{a}}+\sigma \mathrm{d} \alpha_{\mathrm{i}} \\
-\alpha_{\mathrm{i}} \mathrm{d} \sigma & =S_{\mathrm{a}} \mathrm{d} T_{\mathrm{i}} \\
U_{\mathrm{a}}-\alpha_{\mathrm{i}} \sigma & =T_{\mathrm{i}} S_{\mathrm{a}}
\end{aligned}
$$

where $U_{\mathrm{a}}$ and $S_{\mathrm{a}}$ are the average interfacial energy and entropy per unit volume of the mixture, $\alpha_{i}$ is the amount of interface per unit volume of the mixture and $T_{\mathrm{i}}$ the average temperature of the interface.

It must be stressed that for a bubbly fluid in which the bubbles do not break up or coalesce, the interface density $\alpha_{\mathrm{i}}$ depends on $\alpha_{\mathrm{g}}$ and $\rho_{\mathrm{g}}$ through a relation expressing the constancy of the mass per bubble

$$
\alpha_{i}^{3} \sim \alpha_{g}^{2} \rho_{g} .
$$

Moreover, since we supposed the liquid and the gas to have the same temperature $T$, this implies that the interface temperature is also equal to $T$,

$$
T_{\mathrm{i}}=T \text {, }
$$

and the thermodynamic relations for the interface of a one-temperature dispersion are finally rewritten as

$$
\mathrm{d} U_{\mathrm{a}}=T \mathrm{~d} S_{\mathrm{a}}+\frac{\sigma \alpha_{\mathrm{g}}}{a \rho_{\mathrm{g}}} \mathrm{d} \rho_{\mathrm{g}}+\frac{2 \sigma}{a} \mathrm{~d} \alpha_{\mathrm{g}},
$$

where

$$
\begin{gathered}
S_{\mathrm{a}}=-\alpha_{\mathrm{i}} \frac{\mathrm{d} \sigma}{\mathrm{d} T}, \\
U_{\mathrm{a}}=\alpha_{\mathrm{i}}\left(\sigma-T \frac{\mathrm{d} \sigma}{\mathrm{d} T}\right),
\end{gathered}
$$

and

$$
a=3 \alpha_{\mathrm{g}} / \alpha_{\mathrm{i}}
$$

is the bubble radius which, like $\alpha_{i}$, must be considered as a function of $\alpha_{\mathrm{g}}$ and $\rho_{\mathrm{g}}$.

\section{The kinetic energy.}

Let us consider bubbles with an average centre of mass velocity $\mathbf{V}_{\mathbf{g}}$ flowing through a liquid with average velocity $\mathbf{V}_{1}$ and expanding (or shrinking) at a rate $\dot{a}=\mathrm{d} a / \mathrm{d} t$. It was shown above that, for low enough $\dot{a}$ and $\ddot{a}$, the microgradients of $\tilde{\rho}_{1}^{0}$ and $\tilde{\rho}_{\mathbf{g}}^{0}$ were negligible. 
For such a mixture, the kinetic energy per unit volume only involves the mean velocities and their squared fluctuations, and we can write it as

$$
E_{\text {kin }}=\frac{1}{2} \rho_{\mathrm{g}}\left(V_{\mathrm{g}}^{2}+\left(\Delta \widetilde{\mathrm{v}_{\mathrm{g}}}\right)^{2}\right)+\frac{1}{2} \rho_{1}\left(V_{1}^{2}+\left(\Delta \widetilde{v_{1}}\right)^{2}\right)
$$

where $\rho_{\mathrm{g}}$ and $\rho_{1}$ are defined in (22) and where $\left(\Delta \tilde{v}_{\mathrm{k}}\right)^{2}$ stands for the average value of the squared velocity fluctuations in the $\mathbf{k}$ phase.

If we neglect the contribution of any random motion of the bubbles, $\left(\Delta \widetilde{v_{g}}\right)^{2}$ will only involve the bubble internal velocity field (2), with the result

$$
\left(\Delta \tilde{v}_{\mathrm{g}}\right)^{2}=\frac{3}{5} \dot{a}^{2} .
$$

This bubble internal motion is transmitted to the surrounding fluid which, when supposed incompressible, will acquire a kinetic energy in the form

$$
\left(\Delta \tilde{v}_{1}\right)^{2}=q\left(\alpha_{\mathrm{g}}\right) \dot{a}^{2} .
$$

The phenomenological function $q\left(\alpha_{g}\right)$ is unknown except in the dilute limit where the liquid velocity field is given by (10) and leads to

$$
\lim _{\alpha_{\mathrm{g}} \rightarrow 0} q\left(\alpha_{\mathrm{g}}\right)=3 \alpha_{\mathrm{g}} .
$$

If we neglect all effects linked to the average relative velocity $\mathbf{V}_{\mathbf{g}}-\mathbf{V}_{\mathbf{l}}$, we can let

$$
\mathbf{V}=\mathbf{V}_{\mathbf{g}}=\mathbf{V}_{1}
$$

and we finally get

$$
E_{\mathrm{kin}}=\frac{1}{2} \rho V^{2}+\frac{1}{2} i v^{2}
$$

with

$$
\begin{aligned}
\rho & =\rho_{\mathrm{g}}+\rho_{1} \\
v & =\frac{\dot{a}}{a}
\end{aligned}
$$

and

$$
i=a^{2}\left(\frac{3}{5} \rho_{\mathrm{g}}+q\left(\alpha_{\mathrm{g}}\right) \rho_{1}\right)
$$

The scalar $i$ represents the inertia of the whole mixture in the bubble expansion rate $v$. One can verify that in the dilute limit, when (29) is taken into account, the expression (32) is the continuum analog of (13).

\section{The Gibbs equation for the whole mixture.}

The internal energy and entropy of the bubbly fluid are the sum of contributions coming from the gas, the liquid and the interface

$$
\begin{aligned}
U_{0} & =\rho_{\mathrm{g}} \varepsilon_{\mathrm{g}}+\rho_{1} \varepsilon_{1}+U_{\mathrm{a}} \\
S & =\rho_{\mathrm{g}} s_{\mathrm{g}}+\rho_{1} s_{1}+S_{\mathrm{a}} .
\end{aligned}
$$

We are mainly concerned with the total energy

$$
U=U_{0}+E_{\text {kin }}
$$

and we want to establish the Gibbs equation of the mixture, i.e. the differential form of $U$ as a function of the state variables. Upon inspection of equations (19) and (23), the state variables occurring in the internal energy are $\rho_{\mathrm{g}}, \rho_{1}, S$ and $\alpha_{\mathrm{g}}$ (recalling that $\alpha_{1}=1-\alpha_{\mathrm{g}}$ ). As for the kinetic energy, if the choice of $\rho \mathbf{V}$ seems the most convenient, what other variables shall we use ? The scalar moment of inertia $i$ ? The bubble expansion rate $v$ ? Any other combination ? In fact our former result (11) suggests that the most convenient choice is the scalar moment of momentum of the mixture

$$
m=\frac{\partial E_{\text {kin }}}{\partial v}=i v .
$$

This choice is not only convenient but also physically justified. As already noticed the theory of micromorphic fluids [19], as well as the study of an expanding sphere in a perfect fluid [18], have shown that the scalar moment of momentum plays, for the expansional degree of freedom, the same role as the angular momentum for the rotational degree of freedom of the particles.

With $m$ as a new state variable, the Gibbs equation for the whole mixture, taking (19), (23), and (31) into account, is found to be

$$
\begin{array}{rl}
\mathrm{d} U=\mu_{\mathrm{g}}^{*} \mathrm{~d} \rho_{\mathrm{g}}+\mu_{1}^{*} \mathrm{~d} \rho_{1}+T & \mathrm{~d} S-p^{*} \mathrm{~d} \alpha_{\mathrm{g}}+ \\
& +\mathrm{V} \cdot \mathrm{d} \rho \mathbf{V}+\nu \mathrm{d} m,
\end{array}
$$

where

$$
\begin{aligned}
& \mu_{\mathrm{g}}^{*}=\mu_{\mathrm{g}}-\frac{V^{2}}{2}-\frac{v^{2}}{2} \frac{\partial i}{\partial \rho_{\mathrm{g}}}+\frac{\sigma \alpha_{\mathrm{g}}}{a \rho_{\mathrm{g}}}, \\
& \mu_{1}^{*}=\mu_{1}-\frac{V^{2}}{2}-\frac{v^{2}}{2} \frac{\partial i}{\partial \rho_{1}},
\end{aligned}
$$

and

$$
p^{*}=p_{\mathrm{g}}-p_{1}+\frac{v^{2}}{2} \frac{\partial i}{\partial \alpha_{\mathrm{g}}}-\frac{2 \sigma}{a} .
$$

As compared to a classical mixture (a mixture with components miscible up to the atomic scale) the Gibbs equation of a suspension of spheres with variable radius (" bubbly fluid ") presents two extra terms : the first one is linked to the expansion of one phase in the other (variable $\alpha_{\mathrm{g}}$ ) and the second to the rate of expansion of the dispersed phase (variable $m$ ).

\section{The equations of motion and the entropy production.}

A thermodynamic state of the mixture being defined by the knowledge of $\rho_{\mathrm{g}}, \rho_{1}, U, \rho \mathbf{V}, \alpha_{\mathrm{g}}$ and $m$, in order to describe the evolution in time of a state, we need equations describing the evolution of each variable. The variables obeying a conservation law present no 
problem and we write for them :

$$
\begin{array}{r}
\frac{\partial \rho_{\mathbf{g}}}{\partial t}+\nabla \cdot \rho_{\mathbf{g}} \mathbf{V}=0 \\
\frac{\partial \rho_{1}}{\partial t}+\nabla \cdot \rho_{1} \mathbf{V}=0 \\
\frac{\partial \rho \mathbf{V}}{\partial t}+\nabla \cdot(\rho \overline{\overline{V V}}+\overline{\bar{\pi}})=0 \\
\pi_{i j}=\pi_{j i} \\
\frac{\partial U}{\partial t}+\nabla \cdot(U \mathbf{V}+\mathbf{V} \cdot \overline{\bar{\pi}}+\mathbf{Q})=0
\end{array}
$$

where the no-slip condition (30) is expressed by neglecting the mass diffusion flux in (41) and (42), while $\overline{\bar{\pi}}$ is the momentum flux and $\mathbf{Q}$ is the heat flux.

Since $m$ is a scalar (invariant in any change of frame), the most general equation of motion that satisfies Galilean invariance is

$$
\frac{\partial m}{\partial t}+\nabla \cdot(m \mathbf{V}+\mathbf{r})=c
$$

where $\mathbf{r}$ is the (invariant) flux of $m$ and $c$ is its (invariant) source.

The equation governing the evolution of $\alpha_{\mathrm{g}}$ is obtained as follows. If the bubbles do not break up or coalesce, we can express the constancy of the bubble mass as

$$
\rho_{\mathrm{g}} a^{3} \sim \alpha_{\mathrm{g}}
$$

If the liquid is incompressible, we have also

$$
\rho_{1} \sim 1-\alpha_{\mathrm{g}} .
$$

We then deduce from (41) and (42) the important relation linking the divergence of the velocity to the bubble rate of expansion

$$
\boldsymbol{\nabla} \cdot \mathbf{V}=3 \alpha_{\mathbf{g}} v
$$

with

$$
v=\frac{1}{a}\left(\frac{\partial a}{\partial t}+\mathbf{V} \cdot \nabla a\right)
$$

together with the desired equation for $\alpha_{\mathrm{g}}$

$$
\frac{\partial \alpha_{\mathbf{g}}}{\partial t}+\mathbf{V} \cdot \nabla \alpha_{\mathrm{g}}=3 \alpha_{\mathrm{g}} \alpha_{1} v .
$$

The unknown terms appearing in the equations of motion are deduced from the entropy production. Taking the partial time derivative of each member of the Gibbs equation (37) and eliminating all the time derivatives (except $\partial S / \partial t$ ) with the equations of motion (41) to (45) and (48), we obtain the entropy equation in the form

$$
\begin{aligned}
\frac{\partial S}{\partial t}+\nabla \cdot(S \mathbf{V}+\mathbf{S}) & =-\frac{1}{T}\left[\mathbf{S} \cdot \nabla T+v\left(c-3 \alpha_{\mathbf{g}} \alpha_{1} p^{*}\right)+\right. \\
& +\mathbf{r} \cdot \nabla v+(\overline{\bar{\pi}}-P \overline{\bar{I}}): \overline{\overline{\nabla V}}]
\end{aligned}
$$

In this equation appears the entropy flux $\mathbf{S}$ defined as a function of the previous fluxes by

$$
T \mathbf{S}=\mathbf{Q}-v \mathbf{r} .
$$

The scalar $P$ appearing in (49) is at first defined as the sum

$$
P=-U+\rho_{\mathrm{g}} \mu_{\mathrm{g}}^{*}+\rho_{1} \mu_{1}^{*}+\rho V^{2}+v m+T S .
$$

With the use of the thermodynamic relations (19) and (23) together with the definitions (38) and (39) of the extended chemical potentials and the expression (32) for $i$ we finally get

$$
P=\alpha_{1} p_{1}+\alpha_{\mathrm{g}}\left(p_{\mathrm{g}}-\frac{2 \sigma}{a}\right)+\frac{1}{3} i v^{2} .
$$

We may call $P$ the generalized pressure and we observe that it is made of the usual mean pressure $\alpha_{1} p_{1}+\alpha_{\mathrm{g}} p_{\mathrm{g}}$, together with a contribution from the surface tension and another from the bubble expansion rate.

\section{Comments on the equations of motion.}

Let us first consider the non-dissipative equations. Considering the entropy production (49), they are characterized by

$$
\begin{aligned}
\mathbf{S} & =\mathbf{r}=0 \\
& \text { and } \quad=3 \alpha_{\mathrm{g}} \alpha_{1} p^{*} \\
\pi_{i j} & =P \delta_{i j} .
\end{aligned}
$$

The detailed expression for $P$ was given in (51). For a dilute mixture verifying (29) and $\rho_{\mathrm{g}}^{0} \ll \rho_{1}^{0}$, we get, from (32)

$$
\frac{1}{3} i v^{2} \simeq \alpha_{\mathrm{g}} \rho_{1}^{0} \dot{a}^{2} .
$$

The resulting expression for $P$ is in complete agreement with that found (from exact averaging) by Biesheuvel and Van Wijngaarden [5] for a dilute bubbly fluid.

The result (53) shows that the relative pressure $p^{*}$, defined in (40), is the motor for the evolution of $m$, like $\nabla P$ is the motor for $\rho \mathbf{V}$. Remembering that

$$
m=i\left(\alpha_{\mathrm{g}}, \rho_{\mathrm{g}}, \rho_{1}\right) v
$$

one can write

$$
\frac{\partial m}{\partial t}+\nabla \cdot m \mathbf{V} \equiv i \dot{v}+\alpha_{\mathbf{g}} v^{2}\left(2 i+3 \alpha_{1} \frac{\partial i}{\partial \alpha_{\mathrm{g}}}\right)
$$


with

$$
\dot{v}=\frac{\partial v}{\partial t}+\mathbf{V} \cdot \nabla v .
$$

The non-dissipative equation for $m$, deduced from (45) completed by (52) and (53), can thus be rewritten in the equivalent form

$$
\begin{aligned}
& \frac{i}{3 a^{2} \alpha_{\mathrm{g}} \alpha_{1}} a \ddot{a}+\left(\rho_{1} \frac{\mathrm{d} q}{\mathrm{~d} \alpha_{\mathrm{g}}}+\frac{2 i}{3 a^{2} \alpha_{1}}\right) \frac{\dot{a}^{2}}{2}= \\
&=p_{\mathrm{g}}-p_{1}-\frac{2 \sigma}{a} .
\end{aligned}
$$

One can verify that the dilute limit of the above equation is exactly the Rayleigh-Plesset equation (9), when viscous terms are discarded. This is a nice result if one remembers the simplicity of our initial equation (45).

The non-dilute form (55) of the Rayleigh-Plesset equation relies entirely on the phenomenological function $q\left(\alpha_{\mathrm{g}}\right)$ and its derivative $\mathrm{d} q / \mathrm{d} \alpha_{\mathrm{g}}$. If we except its dilute limit (29) we do not have much information about $q\left(\alpha_{\mathrm{g}}\right)$. The only attempt to consider the influence of neighbouring bubbles on the expansion motion was performed by Nigmatulin [7] who found the relationship

$$
\begin{aligned}
\left(1-1.1 \alpha_{\mathrm{g}}^{1 / 3}\right) a \ddot{a}+\frac{3}{2}\left(1-1.47 \alpha_{\mathrm{g}}^{1 / 3}\right) \dot{a}^{2}= & \\
= & \frac{1}{\rho_{1}^{0}}\left(p_{\mathrm{g}}-p_{1}-\frac{2 \sigma}{a}\right) .
\end{aligned}
$$

And it is again gratifying to see that it corresponds exactly to the thermodynamic expression (55) provided

$$
q\left(\alpha_{g}\right)=3 \alpha_{g}\left(1-1.1 \alpha_{g}^{1 / 3}+O\left(\alpha_{g}\right)\right) .
$$

It would be interesting to verify if the $1 / 3$ exponent found by Nigmatulin with a cellular scheme is confirmed by other types of calculations. However this is outside the scope of this work.

A last by-product of the non-dissipative equations is the velocity of sound in the mixture, which we derive as

$$
C^{2}=\frac{\rho_{\mathrm{g}} C_{\mathrm{g}}^{2}}{\alpha_{\mathrm{g}}^{2} \rho}\left(1-\frac{\omega^{2}}{\omega_{\mathrm{B}}^{2}}\right)
$$

where $C_{\mathrm{g}}$ is the sound velocity in the pure gas phase. We recover the classical dispersion relation $[22,23]$ but with a bubble eigenfrequency $\omega_{\mathrm{B}}$ which takes into account the presence of neighbouring bubbles. Indeed we find

$$
\omega_{\mathrm{B}}^{2}=\frac{9 \rho_{\mathrm{g}} C_{\mathrm{g}}^{2}}{i}
$$

and we deduce that the phenomenological function $q\left(\alpha_{g}\right)$ appearing in the inertia coefficient $i$ can be determined with the experimental values of $\omega_{\mathrm{B}}\left(\alpha_{\mathrm{g}}\right)$.
Let us now turn to the dissipative equations. If we limit ourselves to linear deviations from equilibrium, the entropy production (49) will be positive for

$$
\begin{aligned}
\mathbf{S} & =-k_{\mathrm{T}} \nabla T, \\
\mathbf{r} & =-a^{2} \eta_{4} \nabla v, \\
\overline{\bar{\pi}}-P \overline{\bar{I}} & =-2 \eta \overline{\overline{\nabla V}} \text { so }-\eta_{2} \nabla \cdot \overline{\overline{\mathbf{V I}}},
\end{aligned}
$$

and

$$
c=3 \alpha_{\mathrm{g}} \alpha_{1}\left(p^{*}-\eta_{3} v\right),
$$

provided the thermal conductivity $k_{\mathrm{T}}$ and the four viscosities $\eta$ to $\eta_{4}$ are positive. Notice that we did not write a possible coupling between $\mathbf{S}$ and $\mathbf{r}$ because it was hard to imagine how a temperature gradient could influence the diffusion of the bubble expansion. Moreover a coupling between $\boldsymbol{\nabla} \cdot \mathbf{V}$ and $v$ is meaningful due to the constraint (46).

The four viscosities depend on the fluid shear viscosity $\eta_{1}$, on the gas bulk viscosity $\xi_{\mathrm{g}}$ and on the volume fraction $\alpha_{g}$. Except for $\eta_{4}$, their dilute limit is known. For instance, inspection of the Rayleigh-Plesset equation (9) leads to the conclusion

$$
\lim _{\alpha_{\mathrm{g}} \rightarrow 0} \eta_{3}=4 \eta_{1}+3 \xi_{\mathrm{g}} .
$$

Moreover when one bubble expands in an infinite fluid, the velocity field (2) inside the bubble and the one in the fluid (10) lead to the microstresses

$$
\underline{\underline{\tau_{g}}}=3 \xi_{\mathrm{g}} v \overline{\bar{I}}
$$

and

$$
\tilde{\underline{\underline{\tau}}}_{\underline{1}}(\mathbf{r})=2 \eta_{1} \frac{a^{3}}{r^{3}} v(\overline{\bar{I}}-3 \overline{\overline{n n}})
$$

where $\mathbf{r}=\boldsymbol{r n}$ is the position from the bubble centre. The volume average of the liquid stress tensor is null and the average stress of the mixture is thus

$$
\bar{\tau}=3 \alpha_{\mathrm{g}} \xi_{\mathrm{g}} v \overline{\bar{I}} .
$$

Recalling that $\stackrel{\bar{\tau}}{\tau}=P \overline{\bar{I}}-\overline{\bar{\pi}}$ and taking (46) and (58) into account, we find

$$
\lim _{\alpha_{\mathrm{g}} \rightarrow 0} \eta_{2}=\xi_{\mathrm{g}} .
$$

When the bubble is placed in a velocity gradient we deduce in a similar way

$$
\lim _{\alpha_{\mathrm{g}} \rightarrow 0} \eta=\eta_{1},
$$

and a more detailed calculation of the mean stresses (or the mean entropy production) would lead to the Einstein formula with the famous $(5 / 2) \alpha_{\mathrm{g}}$ term.

It is to be noticed that for dilute solutions the overall entropy production $\Sigma$ (the right-hand side of (49)) can be calculated exactly by an integral over the local entropy production; when the motion is res- 
tricted to an expansion (no velocity gradients) one finds to first order in $\alpha_{\mathrm{g}}$

$$
T \Sigma=3\left(4 \eta_{1}+3 \xi_{\mathrm{g}}\right) \alpha_{\mathrm{g}} v^{2} .
$$

We conclude that it is entirely due to $\eta_{3}$ and not to $\eta_{2}$. In fact the entropy production (63) was often interpreted in the past as the result of an apparent bulk viscosity $\xi$ such that

$$
T \Sigma=\xi(\nabla \cdot \mathbf{V})^{2} .
$$

Taking (46) into account, this led to the conclusion that

$$
\xi=\frac{4 \eta_{1}+3 \xi_{\mathrm{g}}}{3 \alpha_{\mathrm{g}}}
$$

with the unphysical feature of a diverging value when $\alpha_{\mathrm{g}}$ tends to zero $[3,4]$. We maintain that this is a misleading interpretation of the entropy production. The actual bulk viscosity of dilute bubbly fluids is finite (if not null) since, according to (61), it is merely equal to the bulk viscosity of the gas, and the entropy production coming from it is

$$
\xi_{\mathrm{g}}\left(3 \alpha_{\mathrm{g}} v\right)^{2}
$$

that is to say, an order of magnitude less that (63). One must thus make a clear distinction between the viscosity $\eta_{3}$ related to the bubble expansion rate $v$, and the viscosity $\eta_{2}$ related to the mixture expansion rate $\nabla \cdot \mathbf{V}$.

\section{Conclusion.}

We have reconsidered the problem of the continuum mechanical description of a bubbly fluid. We have found it was possible to improve over previous theoretical treatments of the bubble volumetric changes. This was realized with the scalar moment of momentum as the relevant state variable. The use of this "new " variable [19] is not limited to peculiar situations such as the absence of relative velocity or phase changes. We think that the merit of the proposed model is :

i) to give a simple physical interpretation of the Rayleigh-Plesset equation and to suggest a straightforward way of extending it to non-dilute situations ;

ii) to be compatible with all known results in the dilute limit, including those concerning the stress tensor;

iii) to lead to an entropy production where the roles of the various viscosities are clearly exhibited.

For reasons of simplicity we have here discarded the relative velocity and the temperature difference between the bubbles and the liquid, as well as nonspherical deformations of the bubbles. We have already at our disposal worthy models for the temperature difference $[2,12,21]$ and the velocity difference [24]. Combining these results with the above treatment of the bubble expansion is not a problem. In contrast, the description of the departure from sphericity is a real challenge but ... all in good time !

\section{References}

[1] Rayleigh Lord, Philos. Mag. 34 (1917) 94-98.

[2] Plesset, M. S. and Prosperetti, A., in Ann. Rev. Fluid Mech., M. van Dyke and W. G. Vincenti eds., 9 (1977) 145-185.

[3] Taylor, G. I., Proc. R. Soc. London A 226 (1954) 34.

[4] Batchelor, G. K., An introduction to fluid dynamics (Cambridge University Press) 1967, ch. 4.

[5] Biesheuvel, A. and Van Wijngaarden, L., J. Fluid Mech. 148 (1984) 301-318.

[6] Biesheuvel, A., Ph. D. Thesis, Technical University of Twente (1984).

[7] Nigmatulin, R. I., Int. J. Multiphase Flow 5 (1979) 353-385.

[8] Nigmatulin, R. I., Fundamentals of mechanics of heterogeneous media, Nauka, Moscow, 1978.

[9] Ariman, T., Rheol. Acta 9 (1970) 542-549.

[10] Goodman, M. A. and Cowin, S. C., Arch. Rat. Mech. Anal. 44 (1970) 249-266.

[11] Passman, S. L., Int. J. Eng. Sci. 15 (1977) 117-129.

[12] Drumheller, D. S. and Bedford, A., J. Acoust. Soc. Am. 66 (1979) 197-208.

[13] Nunziato, J. W. and Walsh, E. K., Arch. Rat. Mech. Anal. 73 (1980) 285-311.
[14] Drumheller, D. S., KipP, H. E. and Bedford, A., J. Fluid Mech. 119 (1982) 347-365.

[15] Capriz, G. and Cohen, H., Mech. Res. Commun. 10 (1983) 369-367.

[16] Kochin, N. E., Kibel, I. A. and Roze, N. V., Theoretical Hydrodynamics (Wiley, New York) 1964, ch. 7.

[17] Yakimov, Yu. L., Fluid Dynamics 18 (1984) 678-683.

[18] Lhuillier, D., C.R. Hebd. Séan. Acad. Sci., série II 295 (1982) 95-98.

[19] ERINGen, A. C., Int. J. Eng. Sci. 2 (1964) 205-217.

[20] Bedford, A. and Drumheller, D. S., Int. J. Eng. Sci. 21 (1983) 863-960.

[21] Nigmatulin, R. I., Appl. Sci. Res. 38 (1982) 267-289.

[22] Carstensen, F. L. and Foldy, L. L., J. Acoust. Soc. Am. 19 (1947) 981.

[23] Van Wijngaarden, L., in Ann. Rev. Fluid Mech. (Annual Reviews Inc., Palo Alto U.S.A.) 4 (1972) 369-396.

[24] Lhuillier, D., Int. J. Multiphase Flow (1985), to appear. 